\title{
Anomalous dynamic scaling of interfaces in disordered media
}

\author{
Ning-Ning Pang ${ }^{1}$ and Wen-Jer Tzeng ${ }^{2}$ \\ ${ }^{1}$ Department of Physics, National Taiwan University, Taipei, Taiwan, Republic of China \\ ${ }^{2}$ National Center of Theoretical Sciences, Tsing-Hua University, Hsinchu, Taiwan, Republic of China
}

(Received 17 September 1998)

\begin{abstract}
We study a recently introduced stochastic growth model for interfacial depinning with quenched disorder in $1+1$ dimensions. We numerically investigate the dynamic correlations of the interface roughening process by studying the $q$ th order equal-time height difference correlation functions. We find that this system does not consist of multiscaling behaviors, in contrast to the molecular-beam-epitaxy motivated growth models with annealed noise, although it does exhibit anomalous dynamic scaling and spatiotemporal intermittency. Moreover, we also investigate the influence of different boundary conditions on the global width of the system. For small system sizes, the discrepancy between the obtained global widths with different boundary conditions will moderately alter the value of the roughness exponent. We propose a modified definition of the global width and quantitatively show that this modified definition is more universal for the systems with different boundary conditions and, thus, more applicable to the experimental measurements. [S1063-651X(99)10001-1]
\end{abstract}

PACS number(s): 05.40.-a, 47.55.Mh, 64.60.Ht

\section{INTRODUCTION}

The kinetic roughening phenomenon of growing interfaces in random media has brought about much interest for its widespread applications in nature [1]. Recently, there has been much interest in the scaling behaviors of interfaces roughened by quenched disorder in the media, such as the motion of a domain wall in the random field Ising model [2] and fluid displacement in porous media [3]. The continuum equation to describe the dynamics of such interfaces is given as follows $[4,5]$ :

$$
\partial_{t} h(x, t)=\nu \nabla^{2} h(x, t)+F+\eta(x, h(x, t)) .
$$

Here $h(x, t)$ is the interface height at position $x$ and time $t, F$ is a uniform driving force that pushes the interface advancing, and the random term $\eta(x, h(x, t))$ represents the effect of the quenched disorder in the media, which is Gaussian distributed with zero mean and some short spatial correlation length. Both analytical [6] and numerical [7,8] studies showed that there exists a pinning-depinning transition. Namely, the interface moves with a finite velocity for strong pushing of $F$, while the interface gets pinned after some finite time for small pushing of $F$.

The global interfacial width $w(L, t)$ of the roughened surface obeys the following dynamic scaling form:

$$
w^{2}(L, t) \equiv\left\langle\overline{[h(x, t)-\overline{h(x, t)}]^{2}}\right\rangle \sim L^{2 \chi} f\left(L / t^{1 / z}\right) .
$$

Throughout the paper, the overbar denotes the average over $x$ in the system of size $L$ and angular brackets denote the statistical average. For the correlation length $\xi \sim t^{1 / z} \ll L$, $w(L, t) \sim t^{\beta}$ with $\beta=\chi / z$; for $\xi \sim t^{1 / z} \gg L, w(L, t) \equiv w_{s a t}(L)$ $\sim L^{\chi}$, where $w_{\text {sat }}(L)$ is the saturated global width for systems of size $L . \chi$ and $z$ are known as the roughness exponent and the dynamic exponent, respectively. At the depinning transition, several numerical measurements $[8,9]$ have obtained the roughness exponent $\chi \simeq 1$.2. Growth models with the roughness exponent $\chi>1$ have been coined "superrough" [10], since $w(L, t \rightarrow \infty) / L$ diverges in the thermodynamic limit.
Besides the above standard scaling behavior of the global interfacial width, the most intriguing feature of interfacial advance in superrough growth models is that the global scaling behavior differs substantially from the local scaling behavior. The former is quantified by the measurement of the global width; while the latter is quantified by the measurement of the equal-time height difference correlation function. Das Sarma, Ghaisas, and Kim [10] have hypothesized a generic anomalous dynamic scaling ansatz to describe the behavior of equal-time height difference correlation functions for all superrough growth models. Although this ansatz is not theoretically proven, its applicability to many surfacediffusion-driven nonequilibrium growth models with annealed noise has been numerically verified [11]. Moreover, the study of these nonequilibrium growth models with annealed noise $[11,12]$, proposed in the context of molecularbeam-epitaxy interface growth processes, suggests an intimate link between anomalous dynamic scaling and multiaffinity. This motivates us to study a recently proposed stochastic growth model [13], which is accepted as a cellular automaton described by Eq. (1) in a discrete space-time lattice. In order to know whether the anomalous dynamic scaling ansatz [10] is also applicable to the growth models with quenched noise and whether the system consists of multiscaling behaviors, we investigate these issues by studying the $q$ th order equal-time height difference correlation functions. Subsequently, we want to understand whether different boundary conditions have much influence on the global width of the system. If so, the discrepancy among the experimentally measured values of the roughness exponent, in the literature, might be partially due to this effect. Since it is difficult to fully control and know exactly the boundary conditions in experimental setups, we are motivated to find a better definition of the global width so that it excludes the influences of different boundary conditions and, thus, is more applicable to experimental measurements.

\section{ANOMALOUS DYNAMIC SCALING}

The definition of the growth model [13] in $1+1$ dimensions is as follows: (1) Each site on a square lattice is as- 
signed a random noise $\eta(x, h)$, taking the value 1 with probability $p$ or -1 with probability $q=1-p$. (2) The interface is represented by a set of integers $h(x, t), x=1,2, \ldots, L$. The flat initial conditions, i.e., $h(x, t=0) \equiv 0$ for all $x$, and periodic boundary conditions, i.e., $h(L+1, t) \equiv h(1, t)$ and $h(0, t) \equiv h(L, t)$, are imposed. (3) At each time step $t$, the interface is updated simultaneously for all $x$ :

$$
h(x, t+1)= \begin{cases}h(x, t)+1 & \text { if } v_{x}>0, \\ h(x, t) & \text { otherwise }\end{cases}
$$

in the above expression, the value $v_{x}$ is defined as

$$
v_{x}=h(x+1, t)+h(x-1, t)-2 h(x, t)+g \eta(x, h) .
$$

The parameters $g$ and $q-p$ represent the relative strength of the random pinning force compared to the surface tension and the driving force, respectively.

We are interested in the $q$ th order equal-time height difference correlation function, which is defined as

$$
C_{q}(x, t) \equiv\left\langle\overline{\left[h\left(x_{0}+x, t\right)-h\left(x_{0}, t\right)\right]^{q}}\right\rangle^{1 / q}
$$

with the overbar denoting the spatial average and $\langle\cdots\rangle$ as the statistical average. Thus, the usual equal-time height difference correlation function is given by

$$
G(x, t) \equiv\left[C_{2}(x, t)\right]^{2} .
$$

For superrough growth models, the behavior of $C_{q}(x, t)$, in the regime where the correlation length $\xi \sim t^{1 / z} \ll L$, has been proposed to obey the following anomalous dynamic scaling ansatz $[10,12]$ :

$$
C_{q}(x, t)=x^{\chi_{q}} f_{q}\left(x / t^{1 / z}\right),
$$

where the scaling function $f_{q}(y)$ obeys

$$
f_{q}(y)= \begin{cases}y^{-\kappa_{q}} & \text { for } y \ll 1 \\ y^{-x_{q}} & \text { for } y \gg 1\end{cases}
$$

In contrast, for the usual dynamic scaling behavior, the scaling function goes to a constant quickly in the small $x / t^{1 / z}$ limit. Thus, the nonsaturation of the scaling function $f_{q}(y)$ in the small $y$ limit, the signature of anomalous dynamic scaling, gives rise to the difference between global and local scaling behaviors. Then, the $q$ th moment of the step size distribution $C_{q}(1, t)$ does not saturate quickly, either; but it increases with time out to substantially long times. Quantitatively, from Eqs. (7) and (8), we see that, in the interval $1 \leqslant x \ll t^{1 / z}$ at a fixed time slice $t, C_{q}(x, t) \sim x^{\chi_{q}^{\prime}}$ with $\chi_{q}^{\prime}$ $=\chi_{q}-\kappa_{q}$, and, in the regime $1 \ll t^{1 / z} \ll L, C_{q}(1, t) \sim t^{\kappa_{q} / z}$. If the values of the scaling exponents $\left(\chi_{q}^{\prime}\right.$ or $\left.\kappa_{q}\right)$ depend on the moments $q$, the system then displays multiscaling behaviors.

In the following, we undertake extensive numerical studies of various correlation functions of the system. From the experimental point of view, they are more interesting and accessible quantities to be compared with the experimental data.

We first measure the equal-time height difference correlation function $G(x, t)$. The simulation is done with the system size $L=262144$ at $p=p_{c} \simeq 0.8004$ (for $g=1$ ) [13] and

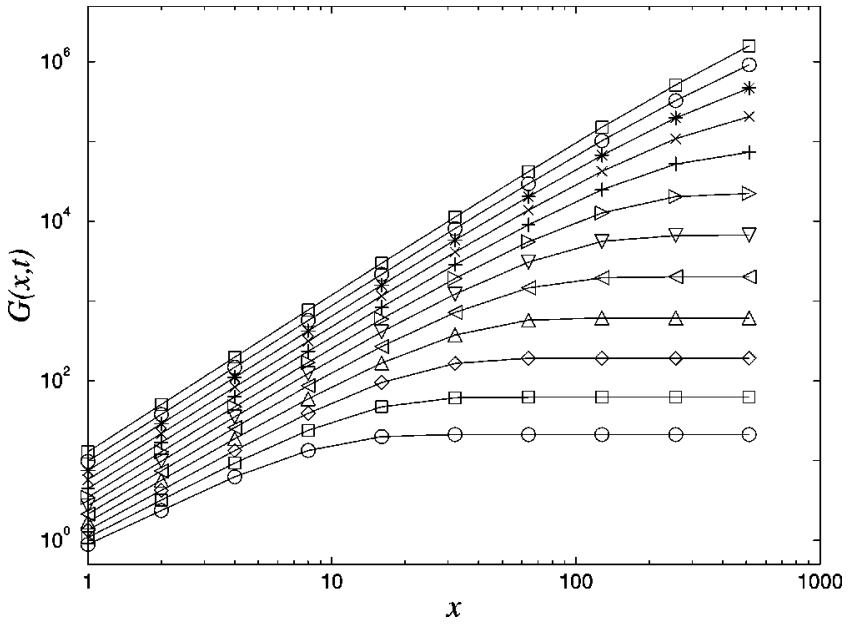

FIG. 1. The log-log plot of a set of $G(x, t)$ vs $x$ for times $t$ $=32,64, \ldots, 65536$ steps (from bottom to top), at the depinning transition $p=p_{c}$. The data are obtained from 100 independent runs with the system size $L=262144$.

averaged over 100 realizations. Figure 1 is the $\log -\log$ plot of a set of $G(x, t)$ vs $x$ for times $t=32,64, \ldots, 65536$ steps (from bottom to top), at the depinning transition $p=p_{c}$. We then obtain, in Fig. 2, excellent data collapse of the scaled $G(x, t) / x^{2 \chi}$ vs $x / t^{1 / z}$ for times $t=32,64, \ldots, 65536$ steps, at the depinning transition $p=p_{c}$, by inserting the values of the roughness exponent $(\chi=1.23)$ and the dynamic exponent $(z=1.42)$. The nonsaturation of the scaling function in Fig. 2 , in the regime $x \ll t^{1 / z} \ll L$, gives strong evidence that the equal-time height difference correlation function $G(x, t)$ obeys the anomalous dynamic scaling ansatz, instead of the usual simple dynamic scaling. Here, the dynamic exponent $z(=1.42 \pm 0.02)$ is obtained indirectly, through the relation $z=\chi / \beta$, from the direct measurements of the roughness exponent $\chi$ and the early time exponent $\beta$. The roughness exponent $\chi(=1.23 \pm 0.01)$ is obtained from the measurement of the saturated global width $w_{\text {sat }}(L)\left(\sim L^{\chi}\right)$ vs the system size $L$, with the system size $L=8,16, \ldots, 8192$ and the number of realizations $\left(=2^{21} / L\right)$ depending on $L$. The early time

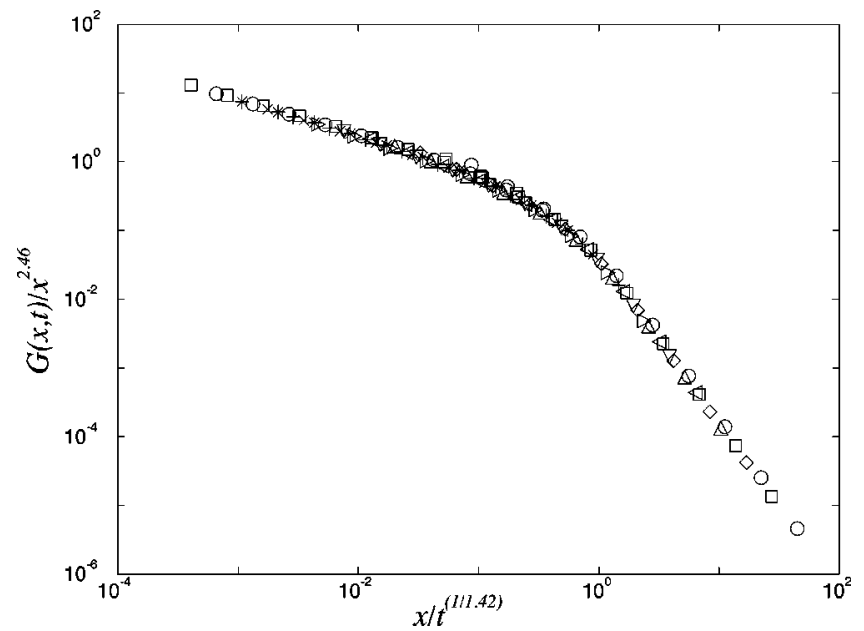

FIG. 2. The data collapse of the scaled $G(x, t) / x^{2 \chi}$ vs $x / t^{1 / z}$ for times $t=32,64, \ldots, 65536$ steps, at the depinning transition $p=p_{c}$, with $2 \chi=2.46$ and $z=1.42$. The data are obtained from 100 independent runs with the system size $L=262144$. 


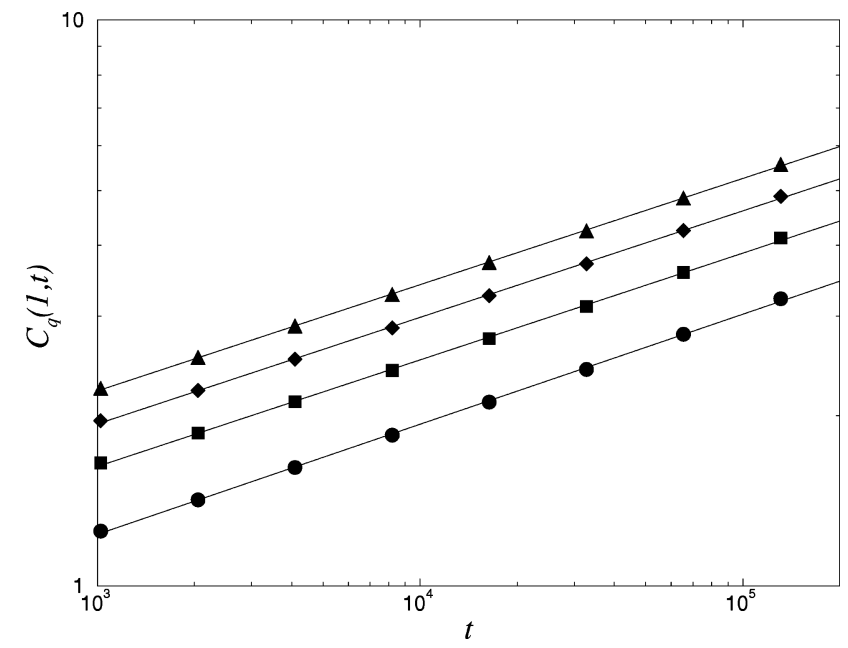

FIG. 3. The log-log plot of the $q$ th moments of the step size distribution $C_{q}(1, t)$ vs time $t$, for $q=1-4$ (from bottom to top), at the depinning transition $p=p_{c}$. The data are obtained from 100 independent runs with the system size $L=262144$. The straight lines, fit by least squares to the data from the interval $1024 \leqslant t$ $\leqslant 131072$, give the exponents $\kappa_{1} / z=0.194 \pm 0.004, \kappa_{2} / z=0.188$ $\pm 0.003, \kappa_{3} / z=0.188 \pm 0.003$, and $\kappa_{4} / z=0.187 \pm 0.003$.

exponent $\beta(=0.865 \pm 0.005)$ is obtained from the measurement of the global width in early times $w(t)\left(\sim t^{\beta}\right)$ vs the time $t \quad(\leqslant 1000$ time steps), with the system size $L=262144$ and averaged over 40 realizations.

Next, we obtain the values of the exponents $\kappa_{q}$ and $\chi_{q}^{\prime}$, by direct measuring $C_{q}(1, t)$ and $C_{q}(x, t)$. Recall that $C_{q}(1, t) \sim t^{\kappa}{ }^{\prime / z}$ in the regime $1 \ll t^{1 / z} \ll L$ and $C_{q}(x, t) \sim x^{\chi_{q}^{\prime}}$ in the interval $1 \leqslant x \ll t^{1 / z}$ at a fixed time slice $t$. Figure 3 shows the log-log plot of the $q$ th moments of the step size distribution $C_{q}(1, t)$ vs time $t$, for $q=1-4$ (from bottom to top), at the depinning transition $p=p_{c}$. The straight lines, fit by least squares to the data from the interval $1024 \leqslant t \leqslant 131072$, give the exponents $\kappa_{1} / z=0.194 \pm 0.004, \quad \kappa_{2} / z=0.188 \pm 0.003$, $\kappa_{3} / z=0.188 \pm 0.003$, and $\kappa_{4} / z=0.187 \pm 0.003$. Figure 4 is the log-log plot of the $q$ th order equal-time height difference correlation function $C_{q}(x, t)$ vs $x$ at $t=1000$ time steps, for $q=1-4$ (from bottom to top), at the depinning transition $p$ $=p_{c}$. The straight lines, fit by least squares to the data from the interval $1 \leqslant x \leqslant 20$, give the exponents $\chi_{1}^{\prime}=0.89 \pm 0.01$, $\chi_{2}^{\prime}=0.89 \pm 0.01, \chi_{3}^{\prime}=0.90 \pm 0.01$, and $\chi_{4}^{\prime}=0.90 \pm 0.01$. The data are obtained from 100 realizations with the system size $L=262$ 144. As a consistency check on the numerical measurements, we obtain $\kappa_{q}+\chi_{q}^{\prime}=1.17 \pm 0.02,1.16 \pm 0.02$, $1.17 \pm 0.02$, and $1.17 \pm 0.02$, respectively, for $q=1-4$, in good agreement with the value $\chi=1.23 \pm 0.01$, extracted from the direct measurement of the global width. The obtained numerical values for $\kappa_{2}$ and $\chi_{2}^{\prime}$ are also in good agreement with an earlier work [14], where $\kappa_{2} \simeq 0.21$ and $\chi_{2}^{\prime}$ $\simeq 0.92$ were obtained by numerical integration of Eq. (1) in $1+1$ dimensions.

The results clearly show that the values of the exponents $\left(\chi_{q}^{\prime}\right.$ and $\left.\kappa_{q}\right)$ are independent of the moments $q$, within the range of statistical uncertainty. Thus, the system does not consist of multiscaling behaviors. Although the stochastic growth models with annealed noise, proposed to mimic the molecular-beam-epitaxy interfacial growth processes [11],

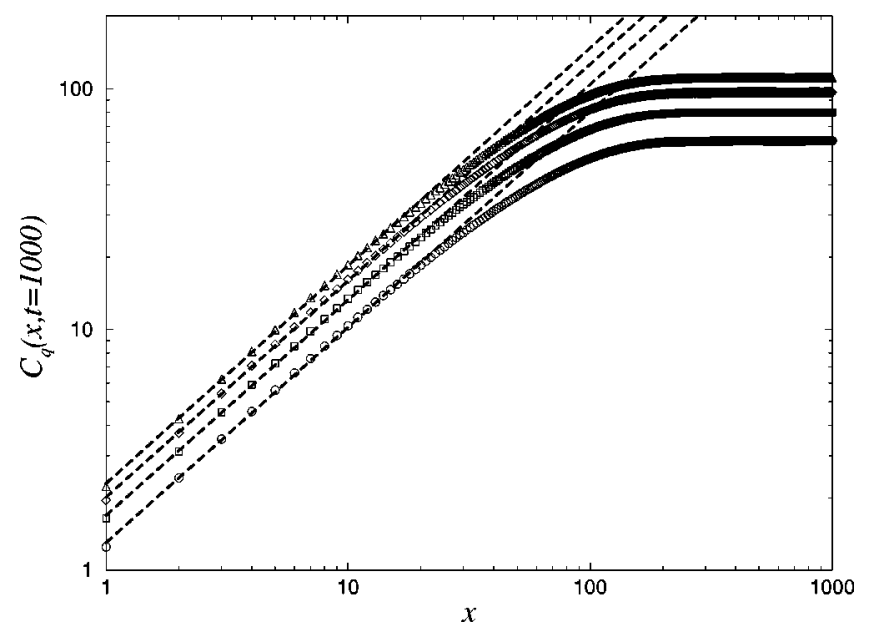

FIG. 4. The $\log -\log$ plot of $C_{q}(x, t)$ vs $x$ at $t=1000$ time steps, for $q=1-4$ (from bottom to top), at the depinning transition $p$ $=p_{c}$. The data are obtained from 100 independent runs with the system size $L=262144$. The straight lines, fit by least squares to the data from the interval $1 \leqslant x \leqslant 20$, give the exponents $\chi_{1}^{\prime}=0.89$ $\pm 0.01, \chi_{2}^{\prime}=0.89 \pm 0.01, \chi_{3}^{\prime}=0.90 \pm 0.01$, and $\chi_{4}^{\prime}=0.90 \pm 0.01$.

have the presence of anomalous dynamic scaling, intermittency, and multiaffinity at the same time, the sufficient conditions to produce multiaffinity in growth models are still unknown, as mentioned in Ref. [11]. Here, we give a concrete example that the presence of anomalous dynamic scaling and spatiotemporal intermittency, which is observed in Ref. [15], does not guarantee the presence of multiaffinity in the system.

\section{BOUNDARY EFFECTS}

All the above simulations are done under the assumptions of flat initial conditions and periodic boundary conditions. It is obvious that the global width of the system $w(L, t)$ is influenced by the imposed boundary conditions. For example, the overall interfacial orientation is, roughly speaking, parallel to the substrate direction, if the periodic boundary conditions are imposed. However, the overall interfacial orientation might not be parallel to the substrate direction, if the free boundary conditions, defined by $h(L+1, t)$ $\equiv h(L, t)$ and $h(0, t) \equiv h(1, t)$, are imposed, instead. Thus, the global width of the latter must be larger than that of the former. However, it is not clear whether this influence is strong enough to alter the roughness exponent or just the prefactor. This motivates us to quantitatively measure the influence of different boundary conditions on the global width of the system. Since the overall interfacial orientation of the system, depending on the boundary conditions, might not be parallel to the substrate direction, we then measure both $w_{\text {sat }}(L)$ and $\widetilde{w}_{\text {sat }}(L)$ for comparison. The former denotes the saturated global width relative to the substrate direction, while the latter denotes the saturated global width relative to the overall interfacial orientation. The definition of $w_{\text {sat }}^{2}(L)\left[\equiv \lim _{t \rightarrow \infty} w^{2}(L, t)\right]$ is already given in Eq. (2). Here, we propose the definition of $\widetilde{w}_{s a t}(L)$ as follows:

$$
\widetilde{w}_{s a t}^{2}(L) \equiv \lim _{t \rightarrow \infty} \widetilde{w}^{2}(L, t) \equiv \lim _{t \rightarrow \infty}\left\langle\overline{\left\langle[h(x, t)-\widetilde{h}(x, t)]^{2}\right.}\right\rangle,
$$




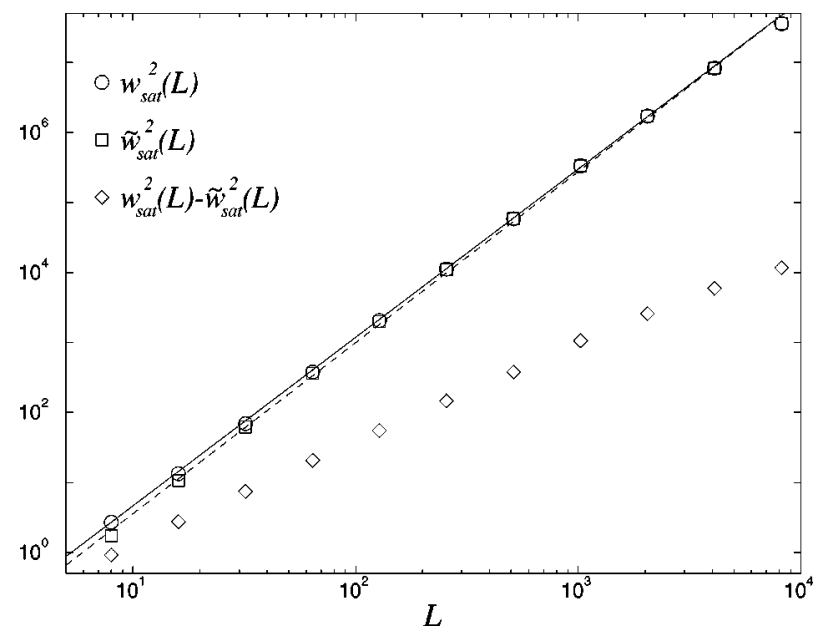

FIG. 5. The log-log plot of $w_{\text {sat }}^{2}(L)$ (circle), $\widetilde{w}_{\text {sat }}^{2}(L)$ (square), and $w_{\text {sat }}^{2}(L)-\widetilde{w}_{\text {sat }}^{2}(L)$ (triangle) vs the system size $L$, with $L$ $=8,16, \ldots, 8192$, at the depinning transition $p=p_{c}$. The number of realizations is equal to $2^{21} / L$ and the simulation is done under free boundary conditions. The straight lines, fit by least squares to $w_{\text {sat }}^{2}(L)$ and $\widetilde{w}_{\text {sat }}^{2}(L)$, give the roughness exponent $\chi=1.20 \pm 0.01$ and $1.22 \pm 0.02$, respectively.

where, as mentioned before, the overbar and angular brackets denote the average over $x$ in the system of size $L$ and the statistical average, respectively. $\widetilde{h}(x, t)$ denotes the heights, measured from the substrate, of a straight line fit by least squares to the interfacial heights of the whole system at a given time $t$. Namely,

$$
\widetilde{h}(x, t)=\overline{h(x, t)}+(x-\bar{x}) s(L, t)
$$

with the slope

$$
s(L, t)=\frac{12}{\left(L^{2}-1\right)} \overline{(x-\bar{x}) h(x, t)},
$$

obtained from the least-squares fit to the interfacial configuration of the whole system at a given time $t$. After some straightforward calculation, we obtain

$$
w_{\text {sat }}^{2}(L)-\widetilde{w}_{\text {sat }}^{2}(L)=\frac{\left(L^{2}-1\right)}{12} \lim _{t \rightarrow \infty}\left\langle s^{2}(L, t)\right\rangle .
$$

Consequently, the difference between these two definitions of the saturated global width is proportional to the magnitude of the slope of the interfacial configuration at the saturated regime.

We subsequently perform the simulation with flat initial conditions and different boundary conditions. Under periodic boundary conditions, the discrepancy between $w_{\text {sat }}(L)$ and $\widetilde{w}_{s a t}(L)$ is negligible as expected, since the overall interfacial orientation is, roughly speaking, parallel to the substrate direction. In contrast, under free boundary conditions, there does exist some discrepancy between $w_{\text {sat }}(L)$ and $\widetilde{w}_{\text {sat }}(L)$ as shown in Fig. 5, which is the log-log plot of $w_{\text {sat }}^{2}(L)$ (circle), $\widetilde{w}_{s a t}^{2}(L)$ (square), and $w_{\text {sat }}^{2}(L)-\widetilde{w}_{s a t}^{2}(L)$ (triangle) vs the system size $L$, with $L=8,16, \ldots, 8192$, at the depinning transition $p=p_{c}$. The number of realizations is equal to $2^{21} / L$ and the simulation is done under free boundary conditions. Although the relevance of the discrepancy between $w_{\text {sat }}(L)$ and $\widetilde{w}_{\text {sat }}(L)$ gradually diminishes as the system size increases, its value $\left\{\equiv\left[w_{\text {sat }}^{2}(L)-\widetilde{w}_{\text {sat }}^{2}(L)\right]^{1 / 2} / w_{\text {sat }}(L)\right\}$ still approximately reaches $3 \%$ for the system size $L=4096$, compared with only $0.06 \%$ for the same system size under the periodic boundary conditions. In addition, the straight lines in Fig. 5, fit by least squares to $w_{s a t}^{2}(L)$ and $\widetilde{w}_{s a t}^{2}(L)$, give the roughness exponent $\chi=1.20 \pm 0.01$ and 1.22 \pm 0.02 , respectively. We see that the value of the roughness exponent $\chi(=1.22 \pm 0.02)$, obtained from the measurement of $\widetilde{w}_{s a t}^{2}(L)$ with the free boundary conditions, is in better agreement with the value of $\chi(=1.23 \pm 0.01)$, obtained from the measurement under periodic boundary conditions. Thus, the modified definition of the global width $\widetilde{w}_{s a t}(L)$ is more universal and applicable to the systems with different boundary conditions. This modified definition of the global width is also more useful in experimental measurements, since it is difficult to control the boundary conditions in experimental setups.

\section{CONCLUSION}

In conclusion, we study the recently introduced stochastic growth model [13] for interfacial depinning with quenched disorder in $1+1$ dimensions. We numerically investigate the dynamic correlations of the interface roughening process by studying the $q$ th order equal-time height difference correlation functions. Due to the superrough nature of the system at the depinning transition, this model violates the usual simple dynamic scaling in the sense that the global and local scalings behave substantially different from each other. We find that the height-height correlation function does obey the recently proposed anomalous dynamic scaling ansatz [10], thus affirming the hypothesis on the generality of this ansatz for all superrough growth models. Any analytical treatment on this type of models should take this effect into account. Moreover, in contrast to superrough stochastic growth models with annealed noise, this system does not consist of multiscaling behaviors, although it exhibits anomalous dynamic scaling and spatiotemporal intermittent behavior [15]. This distinct difference indicates that the origin of multiaffinity in nonequilibrium growth models [11] is very subtle and intriguing. It demands much further work to resolve the issue. Finally, we also investigate the influence of different boundary conditions. For very large system sizes, the influence of different boundary conditions on the global width is negligible. However, for small system sizes, the discrepancy between the obtained global widths with different boundary conditions will moderately alter the value of the roughness exponent. Here, we propose a modified definition of the global width, which is the interfacial width relative to the overall interfacial orientation, instead of the substrate direction. Quantitatively, we show that this modified definition of the global width is more universal for the systems with different boundary conditions. Thus, it is more applicable to the experimental measurements.

\section{ACKNOWLEDGMENTS}

The work of N.-N. Pang is supported in part by the National Science Council of the Republic of China under Grant No. NSC 88-2112-M-002-017. 
[1] See, for example, A.-L. Barabási and H. E. Stanley, Fractal Concepts in Surface Growth (Cambridge University Press, Cambridge, 1995); T. Halpin-Healy and Y.-C. Zhang, Phys. Rep. 254, 215 (1995); J. Krug, Adv. Phys. 46, 139 (1997).

[2] H. Ji and M. Robbins, Phys. Rev. B 46, 14519 (1992).

[3] N. Martys and M. Robbins, Phys. Rev. B 44, 12294 (1992).

[4] R. Bruinsma and G. Aeppli, Phys. Rev. Lett. 52, 1547 (1984).

[5] J. Koplik and H. Levine, Phys. Rev. B 32, 280 (1985).

[6] T. Nattermann, S. Stepanow, L.-H. Tang, and H. Leschhorn, J. Phys. II 2, 1483 (1992).

[7] G. Parisi, Europhys. Lett. 17, 673 (1992).
[8] S. Roux and A. Hansen, J. Phys. I 4, 515 (1994).

[9] H. A. Makse and L. A. N. Amarel, Europhys. Lett. 31, 379 (1995).

[10] S. Das Sarma, S. V. Ghaisas, and J. M. Kim, Phys. Rev. E 49, 122 (1994).

[11] S. Das Sarma, C. J. Lanczycki, R. Kotlyar, and S. V. Ghaisas, Phys. Rev. E 53, 359 (1996).

[12] J. Krug, Phys. Rev. Lett. 72, 2907 (1994).

[13] H. Leschhorn, Physica A 195, 324 (1993).

[14] J. M. López and M. A. Rodríguez, J. Phys. I 7, 1191 (1997).

[15] N.-N. Pang and N. Y. Liang, Phys. Rev. E 56, 1461 (1997). 